\title{
Comment un antigène de surface, le CD38, est-il impliqué dans la sécrétion de l'insuline induite par le glucose?
}

Un tout récent article de l'équipe de H. Okamoto (Sendai, Japon) attire l'attention sur le rôle physiologique et pathologique d'un mécanisme encore mal connu (et controversé) du contrôle de la sécrétion de l'insuline par les cellules pancréatiques des îlots de Langerhans. La libération du calcium stocké dans le réticulum endoplasmique représente l'événement biochimique majeur qui relie le flux glycolytique à la stimulation de la sécrétion d'insuline. Cependant, il existe deux voies possibles de mobilisation du calcium intracellulaire: l'une emprunte la voie de l'inositol 1,4,5-trisphosphate (IP3) alors que la seconde voie fait intervenir l'un des métabolites du $\mathrm{NAD}^{+}$, l'ADP-ribose cyclique (ADPRc). Cette équipe avait déjà proposé, en 1993, à partir de résultats obtenus à l'aide d'un système acellulaire de microsomes isolés des îlots de Langerhans, que c'est par l'intermédiaire de l'ADPRc que l'élévation de la concentration du glucose provoquait, dans les cellules $\beta$ pancréatiques, la mobilisation du calcium [1, 2], établissant le lien entre la concentration de ce métabolite et le métabolisme du glucose. Elle clona l'ADNc de la cyclase pancréatique à partir d'insulinomes humains sur la base d'une analogie surprenante avec l'antigène CD38 présent à la surface des lymphocytes humains; cet antigène possède la double activité ADP-ribosyl cyclase (synthèse de l'ADPRc) et ADPRc-hydrolase (dégradation) [3]. Après surexpression de l'ADNc correspondant dans des lignées immortalisées, on montra que l'activité enzymatique était localisée dans les microsomes et que l'ADPRc s'accumulait bien en pré- était établi le lien entre l'ATP engendré par une élévation du métabolisme du glucose dans les cellules $\beta$ et l'inhibition de l'activité hydrolase, permettant l'accumulation de l'ADPRc. Enfin, la corrélation entre l'activité CD38 produite chez l'animal et la sécrétion de l'insuline fut étudiée à l'aide de souris transgéniques surexprimant CD38 en analysant d'une part, dans les îlots, la mobilisation du calcium en fonction de la concentration du glucose et, d'autre part, la sécrétion de l'insuline au cours de tests de tolérance au glucose chez l'animal entier [4]: en présence d'une concentration élevée de glucose, la mobilisation calcique était multipliée par 3 comparée à celle des témoins, l'insulinémie dans le test de tolérance au glucose était doublée. Ces expériences suggéraient fortement une interrelation entre la voie de l'ADPRc et la sécrétion de l'insuline et excluaient ou minimisaient un rôle de la voie de l'IP3 dans la mobilisation calcique et la sécrétion d'insuline dans les cellules $\beta$ pancréatiques normales.

Deux arguments permettaient de relier certaines formes de diabètes aux mécanismes moléculaires nouvellement décrits: (1) l'expression de CD38 est diminuée dans les îlots de Langerhans de rats dépourvus de glucokinase (contrôlant la première étape du métabolisme glucidique) et dans ceux de souris $o b / o b$; (2) un anticorps monoclonal dirigé contre CD38 et inhibant son activité enzymatique empêche l'accumulation d'ADPRc par les cellules $\beta$ pancréatiques et la libération d'insuline. $\mathrm{Ne}$ devait-on pas rechercher la présence d'auto-anticorps anti-CD38 chez les diabétiques [5] ?
L'intérêt du nouvel article publié dans le Journal of Clinical Investigation [6] réside dans l'étude du système chez 377 diabétiques non insulinodépendants (NIDDM). Une proportion importante de patients $(13,8 \%)$ possède des anticorps sériques dirigés contre CD38 capables, à la fois, d'inhiber l'activité l'ADP ribosyl cyclase, d'activer l'activité hydrolase et d'inhiber la sécrétion d'insuline en réponse au glucose par les cellules $\beta$ pancréatiques d'îlots de rat. Ce résultat est tout à fait en accord avec le modèle décrit précédemment, faisant de l'ADPRc le second messager déclenchant la sécrétion d'insuline et montre que l'équilibre entre les deux activités enzymatiques est fondamental pour la transmission du signal émis par le glucose. Par ailleurs, cette étude a montré une corrélation entre la présence des anticorps anti-CD38 chez les patients et l'évolution de la maladie. Ainsi la majorité des patients NIDDM pour lesquels la glycémie peut être contrôlée par les agents hypoglycémiants seuls ont une concentration d'anticorps anti-CD38 beaucoup plus faible que ceux pour lesquels le contrôle de la glycémie exige en outre le respect d'un régime alimentaire sévère. La concentration d'anticorps est étroitement liée à la potentialité de mobiliser le calcium intracellulaire: les antisérums anti-CD38 sont bien capables d'inhiber la sécrétion d'insuline dépendante du glucose à partir d'explants d'îlots sans affecter la sécrétion basale de l'insuline (qui dépend de l'entrée de calcium extracellulaire). Dans ce cas l'ajout de protéine CD38 recombinante est capable de restaurer la sécrétion dépendante du glucose. Il semble, en outre, que 
des mutations dans le gène codant pour CD38 aient été trouvées chez des patients NIDDM japonais.

Il faut ajouter que les anticorps sont également retrouvés chez $10,1 \%$ des diabétiques insulinodépendants; il est possible, par conséquent, que CD38 agisse comme un auto-antigène dans l'insulite auto-immune des diabètes de type 1 .

M.R.
1. Takasawa S, Tohgo A, Noguchi N, et al. Synthesis and hydrolysis of cyclic ADP-ribose by human leukocyte antigen CD38 and inhibition of the hydrolysis by ATP. J Biol Chem 1993; 268 : 26052-4.

2. Takasawa S, Nata K, Yonekura H, Okamoto H. Cyclic ADP-ribose in insulin secretion from pancreatic beta cells. Science 1993; 259: 370-3.

3. Howard M, Grimaldi JC, Bazan JF, et al. Formation and hydrolysis of cyclic ADP-ribose catalyzed by lymphocyte antigen CD38. Science 1993; 262: 1056-9.

4. Kato I, Takasawa S, Akabane A, et al. Regulatory role of CD38 (ADP-ribosyl cyclase/cyclic ADP- ribose hydrolase) in insulin secretion by glucose in pancreatic beta cells. Enhanced insulin secretion in CD38-expressing transgenic mice. J Biol Chem 1995; 270 : 30045-50

5. Takasawa S, Akiyama T, Nata K, et al. Cyclic ADP-ribose and inositol 1,4,5-trisphosphate as alternate second messengers for intracellular $\mathrm{Ca}^{+}$ mobilization in normal and diabetic beta-cells. $J$ Biol Chem 1998; 273: 2497-500.

6. Ikehata F, Satoh J, Nata K, et al. Autoantibodies against CD38 (ADP-ribosyl cyclase/cyclic ADPribose hydrolase) that impair glucose-induced insulin secretion in noninsulin-dependent diabetes patients. J Clin Invest 1998; 102: 395-401.

\section{BRÈVES}

L'ostéoprotégérine et son ligand: un couple important pour le métabolisme osseux. Les ostéoclastes, dont la fonction est de résorber la matrice osseuse, dérivent des cellules souches hématopoïétiques (alors que les ostéoblastes, qui synthétisent les constituants osseux, ont une origine différente) et ont un ancêtre commun avec les monocytes-macrophages [1]. Leur identification repose sur plusieurs critères: leur expression du récepteur de la calcitonine, une activité enzymatique phosphatase acide tartrate-résistante, et leur capacité à résorber des particules osseuses in vitro; cette dernière propriété étant la base du test fonctionnel identifiant les ostéoclastes. Leur différenciation in vitro à partir de progéniteurs hématopoïétiques requiert la présence de cellules stromales et de vitamine D3, mais on ne connaissait pas la (les) molécules responsable(s) de cette différenciation terminale. En 1997, l'ostéoprotégérine (OPG), structurellement proche de la famille des récepteurs du TNF (tumor necrosis factor) a été identifiée comme un inhibiteur naturel de l'activité des ostéoclastes et de leur différenciation [2]. Le groupe d'AMGEN a alors suspecté que l'OPG agissait en séquestrant le facteur responsable de la différenciation des ostéoclastes, le rendant ainsi indisponible. La structure de l'OPG prédisait que son ligand potentiel devait être une protéine transmembranaire (de type II) de la famille du TNF, ce que confirme une publication récente dans Cell
[3]. La stratégie logique pour identifier ce ligand consistait à utiliser comme sonde en immunofluoresence une protéine de fusion OPGFc à la recherche de cellules exprimant le ligand à leur surface, et une lignée de cellules hématopoïétiques murines, 32D, s'est révélée positive. L'ADNc codant pour le ligand d'OPG (OPG-L) murin a ensuite été cloné par expression à partir des cellules 32D et l'ADNc codant pour l'OPG-L humain à partir d'une banque de tissu ganglionnaire. De masse moléculaire $45 \mathrm{kDa}$, transmembranaire (un fragment de $31 \mathrm{kDa}$ est relargué sous forme soluble), orienté avec une partie amino-terminale intracytoplasmique, OPG-L est identique à deux protéines récemment décrites comme immunomodulateurs (RANCE et TRANK ligand). Le transcrit d'OPG-L est détecté dans les cellules stromales médullaires, les ganglions, la rate et le thymus (cette expression dans les organes lymphoïdes s'expliquant peut-être par le rôle potentiel de OPG-L dans la régulation de la réponse immune), et les régions osseuses où le métabolisme est intense. In vitro, OPG-L se lie efficacement et spécifiquement à la protéine OPG, confirmant qu'il en est bien le (ou un des) ligand(s). En synergie avec le M-CSF (monocyte colony-stimulating factor ou CSF-1), OPG-L permet la différenciation de précurseurs monocytaires en ostéoclastes fonctionnels et cela même en l'absence de cellules stromales et de vitamine D3 requises antérieurement. OPG$\mathrm{L}$ n'est cependant pas un facteur de croissance à proprement parler car, seul, il est inactif, et son association au M-CSF est indispensable pour assurer la survie et la prolifération des précurseurs monocytaires. OPG-L agit surtout sur la maturation terminale des ostéoclastes ce que traduit, d'une part, l'expression (en association avec le M-CSF) des transcrits spécifiquement associés à cette voie de différenciation (récepteur de la calcitonine, enzymes lysosomiales, intégrine avb3) et, d'autre part, la formation de cellules géantes multinucléées caractéristiques douées d'une activité de résorption osseuse. Celle-ci est mesurée in vitro et in vivo (induction d'une hypercalcémie, ostéopénie confirmée par étude histomorphométrique) après injection de la forme soluble du ligand purifié. L'importance du couple OPG, OPG-L dans le métabolisme osseux est aussi confirmée par l'ostéporose sévère que développent les animaux dont le gène $O P G$ a été invalidé, étude citée dans l'article de Cell mais qui n'est pas encore publiée. Restent à identifier maintenant le récepteur de OPG-L à la surface des précurseurs ostéoclastiques, à clarifier le rôle de cette molécule dans la régulation de la réponse immune, et à en déterminer l'utilité potentielle en thérapeutique.

[1. Jurdic P, Solari F. Med Sci 1997; 13: 1285-93.]

[2. Simonet W, et al. Cell 1997; 89 : 309-19.]

[3. Lacey DL, et al. Cell 1998; 93 : 165-76.] 